\title{
Evaluation of Aerodynamic Force and Stress Properties of Propeller of Model Airplane Using by Finite Element
}

\section{Analysis}

Satoshi Fukui and Masayuki Nishida

Department of Mechanical Engineering, Kobe City College of Technology, Kobe 6512194, Japan

\begin{abstract}
The purpose of this study is to evaluate propeller safety using three-dimensional finite element method analysis software. We concluded that the propeller is safe for flying. Propeller is indispensable for generating the impellent force. Therefore, safety evaluation of propeller is necessary. The object of the analysis is a propeller for model airplane. The propeller material is carbon fiber reinforced plastics and there is a carbon cross in the one side surface of the propeller. Other parts are formed with resin. The forces acting on a propeller include centrifugal force, air resistance, and vibration by mass imbalance. We analyzed centrifugal force and air resistance in this study. We made analysis model of propeller by ANSYS. Results show, that the maximum principal stress due to centrifugal force was 23.0 MPa. In addition, the maximum principal stress due to aerodynamic force was $2.3 \mathrm{MPa}$, and the maximum principal stress due to both forces was $24.0 \mathrm{MPa}$.
\end{abstract}

Key words: CFRP (carbon fiber reinforced plastic), propeller, finite element method, centrifugal force, aerodynamic forces.

\section{Introduction}

Model airplanes are enjoyed as a part of the hobbies of many people, from beginners to professionals. Some hobbyists who enjoy model airplanes attempt to increase the performance of their model airplanes. When a model airplane flies, a strong load acts on the propeller. The load of the airplane concentrates on the propeller in particular and the propeller is exposed to complicated stress states. Above all, air resistance and the centrifugal force contribute to stress on the propeller. The propeller is important for the model airplane to produce driving force. Therefore, the propeller needs a margin safety. In this study, we performed a stress analysis of a propeller used by a pylon race competition model airplane.

\section{Analysis Object}

Fig. 1 shows photograph of the propeller in this study, and Table 1 shows propeller specifications.

Corresponding author: Satoshi Fukui, professor, research fields: design engineering, and material mechanics.
This propeller was developed for a prototype model airplane for pylon racing F3D class [1]. The propeller which is connected directly to the reciprocating engine during the flight competition rotates at $30000 \mathrm{rpm}$. The propeller supports a remarkable load from centrifugal force and aerodynamic force. Mount hole diameter of the propeller is a taper hole of $8 \mathrm{~mm}$ and the section of the wing is twisted. The propeller is made of CFRP (carbon fiber reinforced plastic) and there is a carbon cross of the plain weave in the one side surface. The fiber direction of the carbon cloth has on angle of 45 degrees radially of the wing.

\section{The Aerodynamic Force}

Fig. 2 shows these forces in relation to the wing. Aerodynamic force affects the propeller when the model airplane flies. Aerodynamic force is a resultant force of lift and drag. These forces are expressed in the following formulas. Each variable is shown in Table 2 .

$$
\begin{aligned}
L & =0.5 \rho \cdot u^{2} \cdot s \cdot C_{L} \\
D & =0.5 \rho \cdot u^{2} \cdot s \cdot C_{D}
\end{aligned}
$$




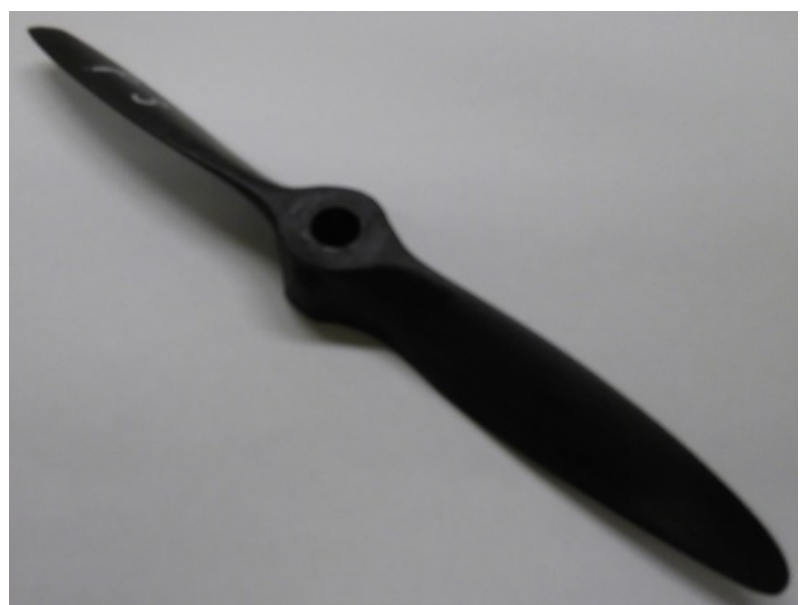

Fig. 1 The shape of propeller.

Table 1 The specifications of the propeller.

\begin{tabular}{ll}
\hline Specification & \\
\hline Maximum radius & $84(\mathrm{~mm})$ \\
Diameter of hole & $8(\mathrm{~mm})$ \\
Width of the wing & $16(\mathrm{~mm})$ \\
Thickness of the wing & $6(\mathrm{~mm})$ \\
Mass & $10.47(\mathrm{~g})$ \\
Material & CFRP \\
\hline
\end{tabular}

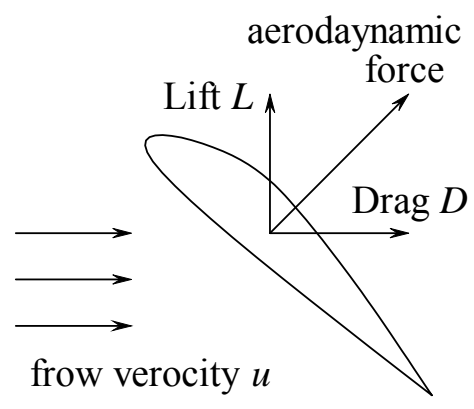

Fig. 2 The relations of the force to act on a wing.

Table 2 Variable of the aerodynamic force estimation.

\begin{tabular}{ll}
\hline Lift & $L(\mathrm{~N})$ \\
Drag & $D(\mathrm{~N})$ \\
Air density Progress speed & $\rho\left(\mathrm{kg} / \mathrm{m}^{3}\right)$ \\
\hline Synthetic velocity & $u(\mathrm{~m} / \mathrm{s})$ \\
Wing area & $s\left(\mathrm{~m}^{2}\right)$ \\
Lift coefficient & $C_{L}$ \\
Drag coefficient & $C_{D}$ \\
\hline
\end{tabular}

We calculated aerodynamic force under conditions shown in Table 3. This propeller rotates at 30,000 rpm and the progress velocity of the model airplane is 75.8 $\mathrm{m} / \mathrm{s}$. This velocity is the record for a competition model aircraft in Japan. We combined the rotational velocity of the propeller with the progress velocity of the airplane to determine synthetic velocity.

We calculated the lift coefficient and drag 
coefficient using software "Wingflow" from the Flow science [3]. This software calculates lift and drag when we input flow velocity and angle of attack into this software. The propeller wing shape resembles the wing shape of "Clark Y" defined in the software. Therefore, we were able to use this software for analysis. However, the size is greatly different. Thus, we applied a Reynolds's law of similarity. When this law executes, drag coefficient and a lift coefficient take the same value each. Therefore, we found a Reynolds number in each section of the propeller using by next formula. In addition, Fig. 3 shows wing shape of "Clark Y" of the software.
We decided velocity to input into software than Reynolds number that we found. In addition, we found the angle of attack as synthetic velocity direction and a relative angle with the torsion angles of the propeller. We input those values softly and demanded lift and drag and we calculated a drag coefficient and a lift coefficient by Eqs. (1) and (2). We calculated lift and drag than those coefficients again and we assumed those resultant force aerodynamic force.

Fig. 4 shows various forces that we calculated them at $1 \mathrm{~mm}$ intervals. Lift and the aerodynamic force change in approximately similar form, and both begin

Table 3 Conditions of the aerodynamic force.

\begin{tabular}{ll}
\hline Progress speed & $75.8(\mathrm{~m} / \mathrm{sec})$ \\
Rotate speed & $30,000(\mathrm{rpm})$ \\
\hline Atmosphere temperature & $298(\mathrm{~K})$ \\
Fluid type & Incompressible fluid \\
Flow type & Laminar flow \\
\hline
\end{tabular}

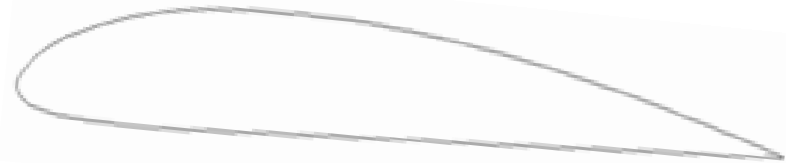

Fig. 3 The wing shape of "Clark Y".

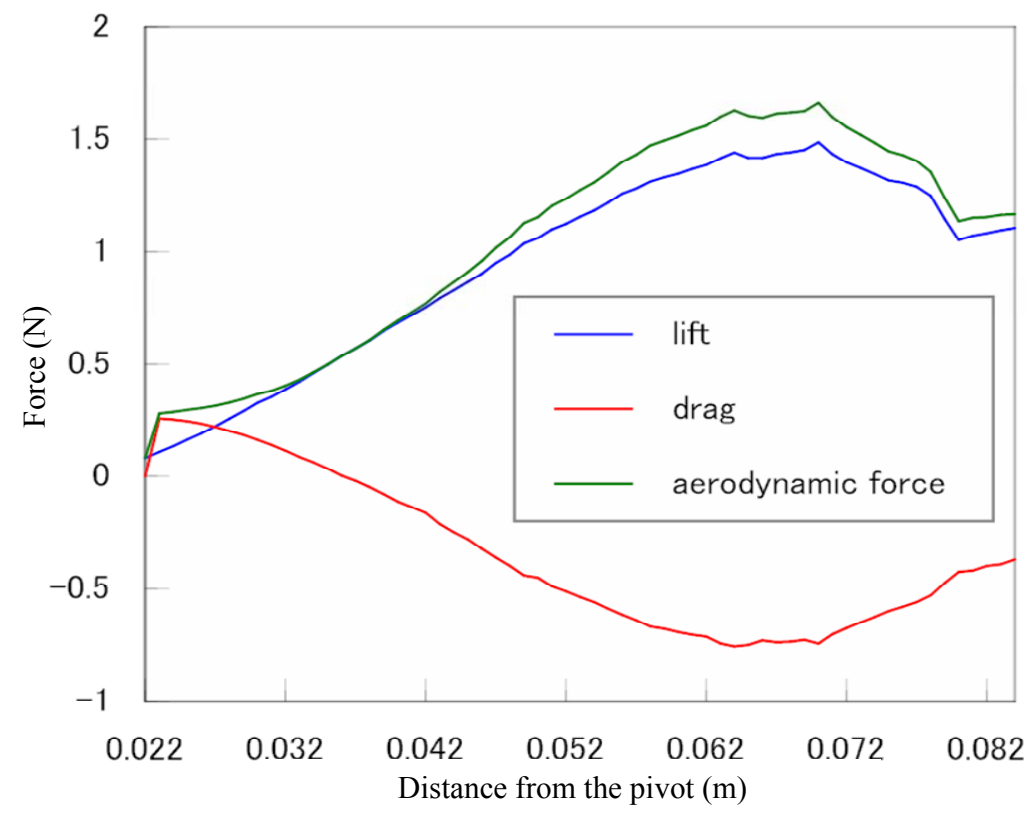

Fig. 4 The results of various forces. 
to decrease from the area beyond $70 \mathrm{~mm}$ from the pivot point. The maxim aerodynamic force calculated by the software was $1.7 \mathrm{~N}$, which was observed on the position of $70 \mathrm{~mm}$ from the axis of rotation. The volume of drag was smaller than lift, but it had a symmetric shape.

\section{Material Properties}

Table 4 shows material properties of the tensile test piece that we need to finite element method analyze. The propeller is made of carbon fiber reinforced plastics. This propeller is composite material and it has anisotropy. Thus, Young's modulus and the Poisson's ratio cannot be obtained. However, The Young's modulus and Poisson's ratio were identified from the result of propeller tensile testing. Fig. 5 shows, the size of the tensile test piece. A tensile test revealed the following results: $11.73 \mathrm{GPa}$ of the Young's modulus; 0.278 of the Poisson's ratio; and $1,267.5 \mathrm{~kg} / \mathrm{m}^{3}$ of the density. In addition, we analyzed assuming this propeller is uniform material and isotropy material.

\section{Stress Analysis}

We used the general-purpose nonlinear finite element method analysis software (ANSYS WorkBench; CYBERNET SYSTEM Co. Ltd.) for the analysis. Fig. 6 shows the analysis model of the propeller. The element types of the model are tetrahedral and the element has 10 nodes. The length of the element is $2 \mathrm{~mm}$. We restricted any movement at the symmetry plane of the model for the analysis and we set the displacement $0 \mathrm{~mm}$.

We analyzed centrifugal force, aerodynamic force,

Table 4 Material properties.

\begin{tabular}{ll}
\hline Density & $1,267.5\left(\mathrm{~kg} / \mathrm{m}^{3}\right)$ \\
Young's modulus & $11.73(\mathrm{GPa})$ \\
Poisson's ratio & 0.278 \\
\hline
\end{tabular}

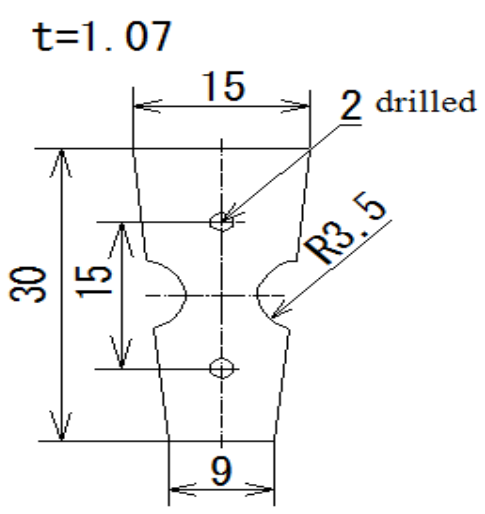

Fig. 5 Shape and size of tensile test piece.

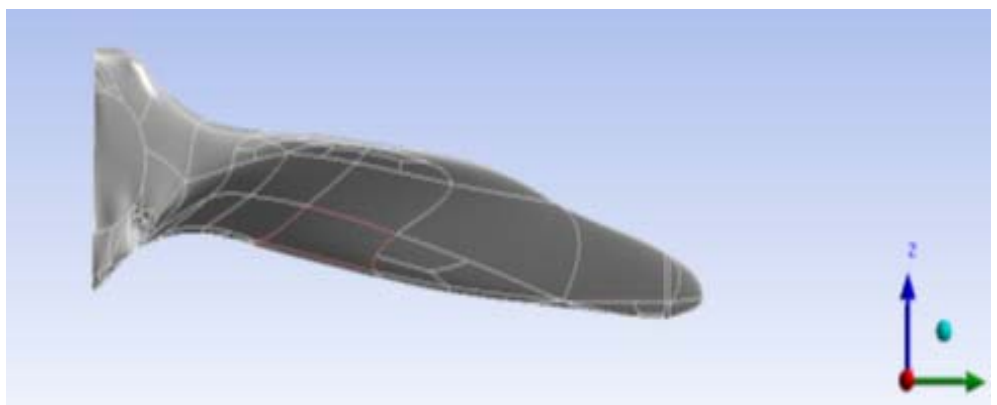

Fig. 6 The propeller used for the analysis model. 
and both types of force working simultaneously. Fig. 7 shows maximum principal stress by centrifugal force. Fig. 8 shows maximum principal stress by aerodynamic force. Fig. 9 shows maximum principal stress by both type of force working simultaneously. Carbon fiber is installed on the surface of each figure on the left. The surface of the right figures is made up of resin.

The left side picture in Fig.7 shows the maximum principal stress of 23.0 MPa that was generated on the left side of the propeller. Likewise, Fig. 8 shows maximum principal stress of $2.30 \mathrm{MPa}$ that was

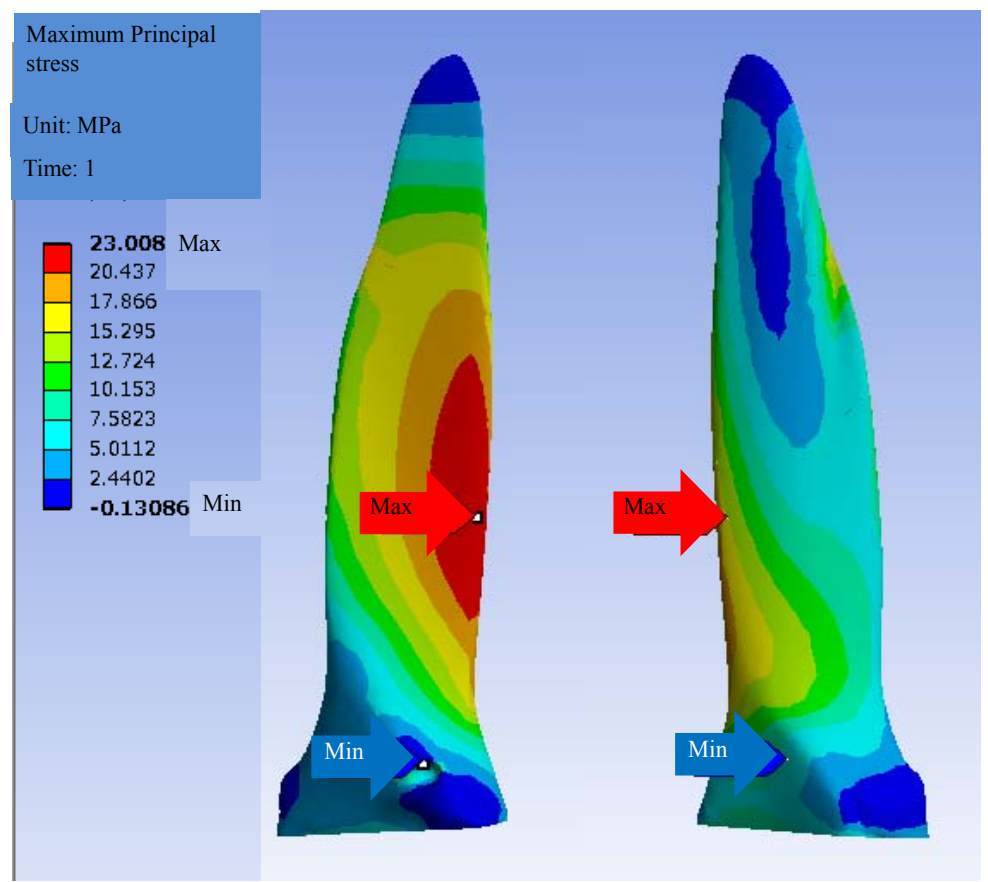

Fig. 7 Maximum principal stress by centrifugal force.

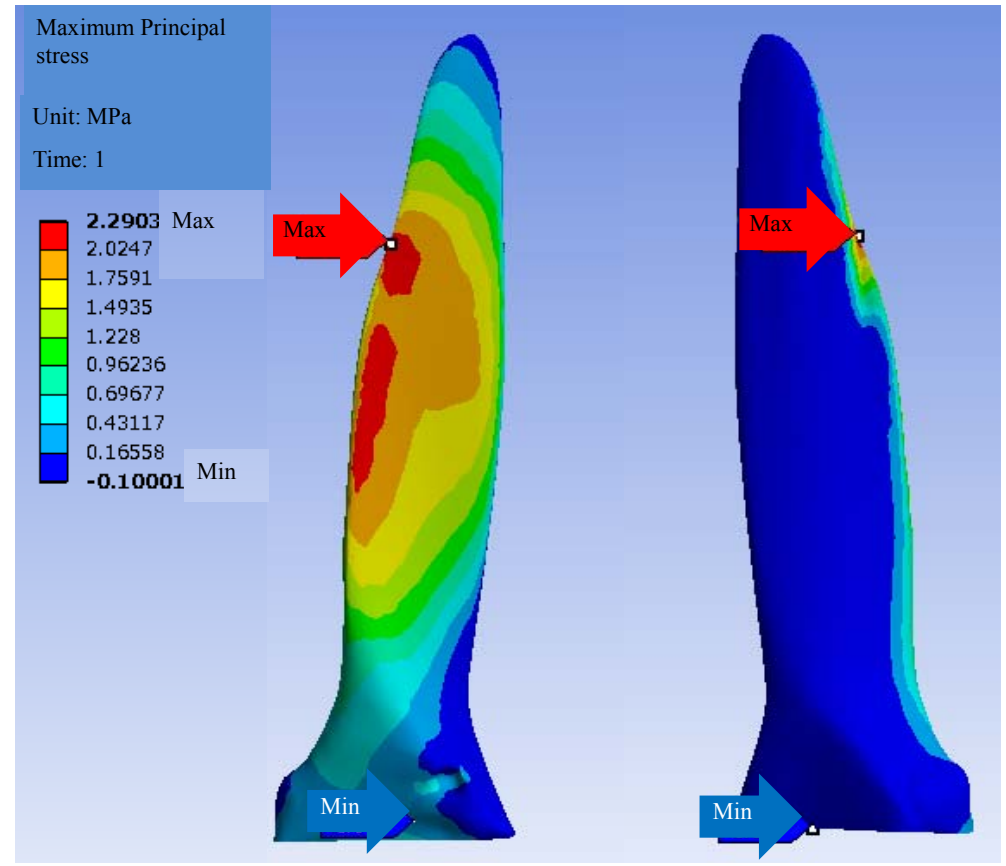

Fig. 8 Maximum principal stress by aerodynamic force. 


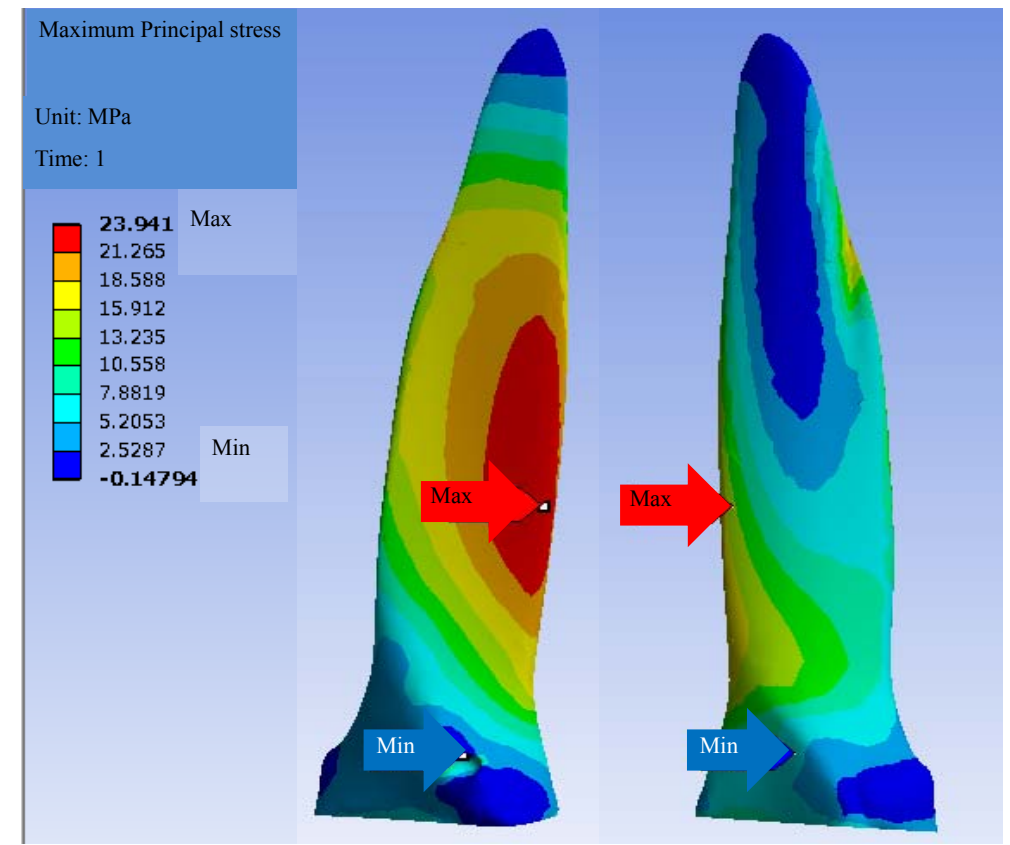

Fig. 9 Maximum principal stress by all forces.

generated on the left side of the propeller. Fig. 9 shows maximum principal stress was $24.0 \mathrm{MPa}$. It occurs on the left side surface of the propeller.

From the analysis results, effect due to the centrifugal force is greater than the aerodynamic force. Therefore, 24.0 MPa was elicited as the final maximum principal stress. Since we know that the fracture stress of this propeller is approximately $120 \mathrm{MPa}$ from the results of the tensile test, the value of 5 was obtained as the safety factor under this condition. In order for FRP materials to be used safely for variable load, such as a model airplane, the value of 4 or more is required for safety factor [4]. Thus, the result indicates the propeller is safe for the model airplane.

\section{Conclusions}

The purpose of this study is to evaluate propeller safety using three-dimensional finite element method analysis software. Results of this study are as follows;

The aerodynamic force calculated by the software was $1.7 \mathrm{~N}$, which was observed on the position of 70 $\mathrm{mm}$ from the axis of rotation. The maximum principal stress analyzed by ANSYS WorkBench was 24.0 MPa, which was observed on the surface of the propeller on the side of the carbon fiber. The data comparison between the centrifugal force and the aerodynamic force revealed that the centrifugal force was greater in load than the aerodynamic force.

\section{References}

[1] Kenji Sumi 2009. "Details of Racing Airplane for F3D." RC Air World Vol.124: 60-3.

[2] Ohashi, H. 1982. Fluid Engineering (1): Corona, Ltd: 6, 127.

[3] Genki Yagawa 2001. Flow Science on Your PC (Wingflow). Kodan Ltd.

[4] Reinforced Plastic Technology Association. 1967. Reinforced Plastic Handbook. Nikkankogyo Shinbun Published Inc. 